\title{
SOME CONDITIONS FOR QUASICONFORMALITY
}

\section{Mihai Cristea}

It is known that if $D, D^{\prime}$ are domains in $\bar{R}^{n}$ and $f: D \rightarrow D^{\prime}$ is a homeomorphism such that the linear dilatation $H(x, f)<H$ for every $x \in D$, then $f$ is quasiconformal. The linear dilatation $H(x, f)$ is defined in a point $x \in D$ such that $x \neq \infty$ and $f(x) \neq \infty$ by $\lim \sup _{r \rightarrow 0}(L(x, f, r) /(l(x, f, r))$, where $L(x, f, r)=\max _{|y-x|=r}|f(y)-f(x)|, l(x, f, r)=\min _{|y-x|=r}|f(y)-f(x)|$. For $0<\alpha<1$ we define $L_{\alpha}(x, f, r)=\max _{|y-x|=\alpha r}|f(y)-f(x)|$ and $H_{\alpha}(x, f)=$ $\lim \sup _{r \rightarrow 0}\left(L_{\alpha}(x, f, r) /(l(x, f, r))\right.$, and it is obvious that $H_{\alpha}(x, f) \leq H(x, f)$. We shall show that if there exist $0<\alpha<1$ and $H>0$ such that $H_{\alpha}(x, f)<H$ for every $x \in D$, then $f$ is $K$-quasiconformal, with $K(\alpha, H, n)=H^{n-1} \alpha^{1-n}$. We shall follow the classical proof of the fact that the metric definition of quasiconformality implies the analytic definition of the quasiconformality, and we shall give a detailed proof for the sake of completeness. We shall use this result to prove that if $f: D \rightarrow D^{\prime}$ is a homeomorphism for which there exist $\varepsilon>0$ and $\delta>0$ such that $M\left(\Gamma_{A}^{\prime}\right)<\delta$ for every ring $A$ in $D$ with $M\left(\Gamma_{A}\right) \leq \varepsilon$, then $f$ is $K$-quasiconformal with $K(\varepsilon, \delta, n)=\left(\exp \left(\omega_{n-1} \varepsilon^{-1}\right)^{1 /(n-1)} / g(\delta)\right)^{n-1}$, where $g$ is a determined function. Therefore $f$ is quasiconformal if and only if $f$ carries rings of small modulus into rings whose modulus is dominated by a constant $\delta$. P. Caraman [1], generalizing a result of Renggli, proved that if $f: D \rightarrow D^{\prime}$ is a homeomorphism with $f$ carrying path families $\Gamma$ from $D$ such that $M(\Gamma)=\infty$ into path families $\Gamma^{\prime}$ such that $M\left(\Gamma^{\prime}\right)=\infty$, then $f$ is quasiconformal. We shall prove that if $f: D \rightarrow D^{\prime}$ is a homeomorphism such that there exists a constant $\delta>0$ such that $M\left(\Gamma^{\prime}\right)>\delta$ for every path family $\Gamma$ from $D$ with $M(\Gamma)=\infty$, then $f$ is quasiconformal. These results (Theorem 2, Theorem 3 and Theorem 4 from our paper) can easily be deduced from Lemma 1 [1], but we give an alternative proof which permits us to evaluate the quasiconformality constant $K$, too.

I wish to thank the referee for his careful reading of the manuscript and his advice. The final form of the present paper was substantially improved by his suggestions.

Throughout the paper, $D, D^{\prime}$ will be domains in $\bar{R}^{n}$ and $f: D \rightarrow D^{\prime}$ a homeomorphism. We shall use the notations and the results from [2]. We denote by $A(r)$ the set of all rings $A=R\left(C_{0}, C_{1}\right)$ in $\bar{R}^{n}$ with the following properties: 1) $C_{0}$ contains the origin and a point $a$ such that $\left.|a|=1.2\right) C_{1}$ contains $\infty$ and a point $b$ such that $|b|=r$. We denote $h(r)=\inf M\left(\Gamma_{A}\right)$ over all rings $A \in A(r)$. Then $h:(0, \infty) \rightarrow(0, \infty)$ is a decreasing function such that $\lim _{r \rightarrow 0} h(r)=\infty$ and 
$\lim _{r \rightarrow \infty} h(r)=0($ see $[2$, Theorem 11.7, p. 34]), and we define $g:(0, \infty) \rightarrow(0, \infty)$, $g(x)=h^{-1}(x)$ for every $x \in(0, \infty)$. Also, if $A=R\left(C_{0}, C_{1}\right)$ is a ring such that $c, \infty \in C_{1}, a, b \in C_{0}$, then $M\left(\Gamma_{A}\right) \geq h((|c-a|) /(|b-a|))$ (see [2, Theorem 11.9, p. 36]). For every set $X \subset R^{n}$, we denote by $m_{p}(X)$ the $p$-dimensional Hausdorff measure of $X$. We denote by $V_{n}=m_{n}\left(B^{n}\right)$ and by $\omega_{n-1}=m_{n-1}\left(S^{n-1}\right)$, where $B^{n}$ is the unit ball from $R^{n}$ and $S^{n-1}$ is the boundary of $B^{n}$. We also set $L(x, f)=\lim \sup _{h \rightarrow 0}|f(x+h)-f(x)| /|h|$.

Lemma 1. Let $f: D \rightarrow D^{\prime}, 0<\alpha<1$ and $h>0$ be such that $H_{\alpha}(x, f)<H$ for every $x \in D$. Then $f$ is $A C L$.

Proof. Let $Q=\left\{x \in R^{n} \mid a_{i} \leq x \leq b_{i}\right\}$ be a cube in $D \backslash\left\{\infty, f^{-1}(\infty)\right\}$ and consider the projection $P: R^{n} \rightarrow R^{n-1}=R_{n}^{n-1}$. For each Borel set $A \subset$ int $P Q$, we set $E_{A}=Q \cap P^{-1}(A)$. Setting $\varphi(A)=m\left(f\left(E_{A}\right)\right)$ we obtain a set function $\varphi$ in int $P Q$. By the Lebesgue theorem, $\varphi$ has a finite derivative $\varphi^{\prime}(y)$ for almost every $y \in \operatorname{int} P Q$. We shall prove that $f$ is absolutely continuous on the segment $J=E_{y}$. Let $F$ be a compact subset of $J \cap$ int $Q$ and let $F_{k}=$ $\left\{x \in F \mid L_{\alpha}(x, f, r) \leq H l(x, f, r)\right.$ for every $\left.0<r<1 / k\right\}$; for $k \in N$. Since $f$ is continuous, each $F_{k}$ is a compact set, $F_{k} \subset F_{k+1}$ and $F=\bigcup_{k=1}^{\infty} F_{k}$. We now choose $k \in N$ such that $1<k d(F, F r Q)$, we fix $k$ and we choose $\varepsilon>0$ and $t>0$. By [2, Lemma 31.1, p. 106], there exists $\delta>0$ such that for every $r \in(0, \delta / \alpha)$ there exists a finite covering of $F_{k}$ with open intervals $\Delta_{1}, \Delta_{2}$, $\ldots, \Delta_{p}$ such that $m\left(\Delta_{i}\right)=2 \alpha r$, the centre of each $\Delta_{i}$ belongs to $F_{k}$, each point of $J$ belongs to at most two $\Delta_{i}, i=1,2, \ldots, p$ and $p \alpha r<m\left(F_{k}\right)+\varepsilon$. We now choose $r<\min (\delta / \alpha, 1 / k)$ such that if $|x-z| \leq 2 \alpha r, x, z \in Q$, it follows that $|f(x)-f(z)|<t$. Let $A_{i}$ be the open $n$-balls whose diameter is $\Delta_{i}, x_{i}$ be the center of $\Delta_{i}$ and $B_{i}$ the open $n$-balls centered at $x_{i}$ and of radius $r, i=1,2, \ldots, p$. Then $B_{i} \subset E_{B}$, where $B=B^{n-1}(y, r)$, and we denote $L_{i}=L_{\alpha}\left(x_{i}, f, r\right)$ and $l_{i}=l\left(x_{i}, f, r\right), i=1,2, \ldots, p$. Since $x_{i} \in F_{k}$, we have $L_{i} \leq H l_{i}, i=1,2, \ldots, p$. Then $F_{k} \subset \bigcup_{i=1}^{p} A_{i}, d\left(f\left(A_{i}\right)\right)<t, i=1,2, \ldots, p$; hence

$$
m_{1}^{t}\left(f\left(F_{k}\right)\right) \leq \sum_{i=1}^{p} d\left(f\left(A_{i}\right)\right) \leq 2\left(\sum_{i=1}^{p} L_{i}\right)
$$

By Hölder's inequality we obtain

$$
m_{1}^{t}\left(f\left(F_{k}\right)\right)^{n} \leq 2^{n}\left(\sum_{i=1}^{p} L_{i}\right)^{n} \leq 2^{n}\left(\sum_{i=1}^{p} 1\right)^{n-1}\left(\sum_{i=1}^{p} L_{i}^{n}\right)
$$




$$
\begin{aligned}
& =2^{n} p^{n-1} \sum_{i=1}^{p} L_{i}^{n} \leq 2^{n} H^{n} p^{n-1} \sum_{i=1}^{p} l_{i}^{n} \\
& \leq 2^{n} H^{n} \alpha^{1-n} r^{1-n}\left(m_{1}\left(F_{k}\right)+\varepsilon\right)^{n-1} \sum_{i=1}^{p} l_{i}^{n} \\
& \leq 2^{n} H^{n} \alpha^{1-n} r^{1-n}\left(m_{1}\left(F_{k}\right)+\varepsilon\right)^{n-1} V_{n}^{-1} \sum_{i=1}^{p} m\left(f\left(B_{i}\right)\right) .
\end{aligned}
$$

Let $q=[1 / \alpha]+1$. Then $q \alpha>1$, and every point from $E_{B}$ belongs to at most $6 q$ balls $B_{i}$. Indeed, let $\bar{y} \in E_{B}$ be such that $\bar{y} \in B\left(x_{j}, r\right)$. If $B\left(x_{i}, r\right) \cap B\left(x_{j}, r\right) \neq$ $\emptyset$, then $\left|x_{i}-x_{j}\right|<2 r$; hence $\bar{y}$ may belong only to a ball $B\left(x_{i}, r\right)$ such that $\left|x_{i}-x_{j}\right|<2 r$. Suppose that we have more than $6 q$ points $x_{i}$ in $J \cap B\left(x_{j}, 2 r\right)$. Let $I=\left\{i \in Z \mid x_{i} \in J \cap B\left(x_{j}, 2 r\right)\right\}$. Then $\sum_{i \in I} m\left(\Delta_{i}\right) \geq 12 q \alpha r>12 r$. Since $m\left(\bigcup_{i \in I} \Delta_{i}\right)<6 r$, it follows that there exists a point $x \in J$ such that $x$ belongs to at least 3 sets $\Delta_{i}$, which represents a contradiction. Then $\bar{y}$ may belong to at most $6 q$ balls $B\left(x_{i}, r\right)$; hence every point of $E_{B}$ may belong to at most $6 q$ balls $B_{i}$. Since every point of $f\left(E_{B}\right)$ belongs to at most $6 q f\left(B_{i}\right)$, we have

$$
\sum_{i=1}^{p} m\left(f\left(B_{i}\right)\right) \leq 6 q m\left(f\left(E_{B}\right)\right)=6 q \varphi(B) .
$$

Since $F_{k} \subset F$, we have

$$
m_{1}^{t}\left(f\left(F_{k}\right)\right)^{n} \leq\left(2^{n+3} H^{n} V_{n-1} q\left(m_{1}(F)+\varepsilon\right)^{n-1} \varphi(B)\right)\left(V_{n} \alpha^{n-1} m(B)\right)^{-1} .
$$

Letting first $r \rightarrow 0$, then $\varepsilon \rightarrow 0$ and then $t \rightarrow 0$, we obtain $m_{1}\left(f\left(F_{k}\right)\right)^{n} \leq$ $C \varphi^{\prime}(y) m_{1}(F)^{n-1}$, where $C=2^{n+3} H^{n} V_{n-1} q \alpha^{1-n} V_{n}^{-1}$. Since $f\left(F_{k}\right) \nearrow f(F)$, we obtain $m_{1}(f(F))^{n} \leq C \varphi^{\prime}(y) m_{1}(F)^{n-1}$, and from [2, Theorem 30.9, p. 101], we see that $f$ is absolutely continuous on $J$.

Lemma 2. Let $f: D \rightarrow D^{\prime}, 0<\alpha<1, H>0$, such that $H_{\alpha}(x, f)<H$ for every $x \in D$. Then $f$ is a.e. differentiable.

Proof. We have $\mu_{f}^{\prime}(x)<\infty$ a.e. (see [2, Theorem 24.2, p. 83]) and, by the theorem of Rademacher and Stepanov, it suffices to show that $L(x, f)<\infty$ a.e. Let $x_{0} \in D$ be such that $x_{0} \neq \infty, f\left(x_{0}\right) \neq \infty$ and $\mu_{f}^{\prime}\left(x_{0}\right)<\infty$. Then there exists $r_{0}>0$ such that $L_{\alpha}\left(x_{0}, f, r\right)<H l\left(x_{0}, f, r\right)$ for $r \leq r_{0}$. For such $r$ we have

$$
V_{n} L_{\alpha}\left(x_{0}, f, r\right)^{n} \leq V_{n} H^{n} l\left(x_{0}, f, r\right)^{n} \leq H^{n} m\left(f\left(B\left(x_{0}, r\right)\right)\right) .
$$

Hence we obtain the following inequality:

$$
\frac{L\left(x_{0}, f, \alpha \cdot r\right)^{n}}{(\alpha \cdot r)^{n}} \leq \frac{H^{n} m\left(f\left(B\left(x_{0}, r\right)\right)\right)}{\alpha^{n} m\left(B\left(x_{0}, r\right)\right)} .
$$

Letting $r \rightarrow 0$, we see that $L\left(x_{0}, f\right)^{n} \leq H^{n} \alpha^{-n} \mu_{f}^{\prime}\left(x_{0}\right)$. 
Theorem 1. Let $f: D \rightarrow D^{\prime}, 0<\alpha<1, H>0$, such that $H_{\alpha}(x, f)<H$ for every $x \in D$. Then $f$ is $K$-quasiconformal, with $K(\alpha, H, n)=H^{n-1} \alpha^{1-n}$.

Proof. From Lemma 1 and Lemma 2, $f$ is ACL and a.e. differentiable. Let $x \in D$ be such that $f$ is differentiable at $x$ and $\left|f^{\prime}(x)\right|=\sup _{|h|=1}\left|f^{\prime}(x)(h)\right|$, $m_{x}=\inf _{|h|=1}\left|f^{\prime}(x)(h)\right|$, and let $\varepsilon>0$ be fixed. Then there exists $\delta_{\varepsilon}>0$ such that for $|h|<\delta_{\varepsilon}$;

$$
\left|f(x+h)-f(x)-f^{\prime}(x)(h)\right| \leq \varepsilon|h|,
$$

hence

$$
\left|f^{\prime}(x)(h)\right|-\varepsilon|h| \leq|f(x+h)-f(x)| \leq\left|f^{\prime}(x)(h)\right|+\varepsilon|h|
$$

for $|h|<\delta_{\varepsilon}$. Let $r=|h|$. Then for $r \leq \delta_{\varepsilon},\left(\left|f^{\prime}(x)\right|-\varepsilon\right) \alpha r \leq L_{\alpha}(x, f, r)$ and $l(x, f, r) \leq\left(m_{x}+\varepsilon\right) r$. We have

$$
\alpha \frac{\left|f^{\prime}(x)\right|-\varepsilon}{m_{x}+\varepsilon} \leq \frac{L_{\alpha}(x, f, r)}{l(x, f, r)}
$$

for $r \leq \delta_{\alpha}$. We see that if $m_{x}=0$ and $\left|f^{\prime}(x)\right| \neq 0$, then $H_{\alpha}(x, f)$ must be $\infty$; hence either $f^{\prime}(x)=0$ or we have $0<\alpha\left|f^{\prime}(x)\right| / m_{x} \leq H_{\alpha}(x, f)<H$. For $m_{x}>0$ we have

$$
\frac{\left|f^{\prime}(x)\right|^{n}}{\left|J_{f}(x)\right|} \leq \frac{\left|f^{\prime}(x)\right|^{n-1}}{m_{x}^{n-1}}
$$

and

$$
\frac{\left|J_{f}(x)\right|}{m_{x}^{n}} \leq \frac{\left|f^{\prime}(x)\right|^{n-1}}{m_{x}^{n-1}}
$$

(see $\left[2,(14.3)\right.$, p. 44]). Hence if $f$ is differentiable at $x$, we obtain $\left|f^{\prime}(x)\right|^{n} \leq$ $H^{n-1} \alpha^{1-n}\left|J_{f}(x)\right|$ and $\left|J_{f}(x)\right| \leq H^{n-1} \alpha^{1-n} m_{x}^{n}$. We now apply Theorem 34.6 from [2, p. 115], to conclude that $f$ is $K$-quasiconformal, with $K(\alpha, H, n)=$ $H^{n-1} \alpha^{1-n}$.

Theorem 2. Let $f: D \rightarrow D^{\prime}$ be such that one of the following conditions is satisfied:

a) There exist $\varepsilon>0$ and $\delta>0$ such that for every ring $A$ in $D$ such that $M\left(\Gamma_{A}\right) \leq \varepsilon$ it follows that $M\left(\Gamma_{A}^{\prime}\right)<\delta$.

b) There exist $\varepsilon>0$ and $\delta>0$ such that for every ring $A$ in $D$ such that $M\left(\Gamma_{A}^{\prime}\right) \leq \varepsilon$, it follows that $M\left(\Gamma_{A}\right)<\delta$.

Then $f$ is $K$-quasiconformal, with

$$
\left.K(\varepsilon, \delta, n)=\left(\exp \left(\omega_{n-1} \cdot \varepsilon^{-1}\right)^{1 /(n-1)}\right) / g(\delta)\right)^{n-1}
$$


Proof. Suppose that Condition b) holds. Let $\alpha \in(0,1)$ be such that $h(\alpha)=$ $\delta, \lambda=\exp \left(\left(\omega_{n-1} \varepsilon^{-1}\right)^{1 /(n-1)}\right)$, and suppose that there exists a point $x \in D \backslash$ $\left\{\infty \cup f^{-1}(\infty)\right\}$ such that $H_{\alpha}(x, f) \geq \lambda$. Then we can find $r>0$ such that $L_{\alpha}(x, f, r) \geq \lambda l(x, f, r)$ and $\bar{B}\left(f(x), L_{\alpha}(x, f, r)\right) \subset D^{\prime}$. We abbreviate $L_{\alpha}(x, f, r)$ to $L_{\alpha}$ and $l(x, f, r)$ to $l$ and let $B=f^{-1}(B(f(x), l)), C=C f^{-1}\left(B\left(f(x), L_{\alpha}\right)\right)$, $A=R(B, C)$. Then $M\left(\Gamma_{A}\right) \geq h(\alpha)=\delta$ and $M\left(\Gamma_{A}^{\prime}\right)=\omega_{n-1}\left(\log \left(L_{\alpha} l^{-1}\right)\right)^{1-n}$. Since $L_{\alpha} / l \geq \exp \left(\left(\omega_{n-1} \varepsilon^{-1}\right)^{1 /(n-1)}\right)$ if and only if $\omega_{n-1}\left(\log \left(L_{\alpha} l^{-1}\right)\right)^{1-n} \leq \varepsilon$, we obtain $M\left(\Gamma_{A}^{\prime}\right) \leq \varepsilon$ and $M\left(\Gamma_{A}\right) \geq \delta$, which represents a contradiction. We proved that $H_{\alpha}(x, f) \leq \lambda$ for every $x \in D$ and that hence, by Theorem $1, f$ is $K$ quasiconformal, with $K(\varepsilon, \delta, n)=\left(\exp \left(\left(\omega_{n-1} \varepsilon^{-1}\right)^{1 /(n-1)} / g(\delta)\right)\right)^{n-1}$. If Condition a) holds, we prove the same thing for $f^{-1}$, and by [2, Corollary 13.3, p. 42], the theorem is proved.

We remark that we have also proved the following theorem:

Theorem 3. Let $f: D \rightarrow D^{\prime}$ be such that one of the following conditions is satisfied:

a) There exist $\varepsilon>0$ and $\delta>0$ such that for every ring $A$ in $D$ such that $M\left(\Gamma_{A}\right) \geq \delta$ it follows that $M\left(\Gamma_{A}^{\prime}\right)>\varepsilon$.

b) There exist $\varepsilon>0$ and $\delta>0$ such that for every ring $A$ in $D$ such that $M\left(\Gamma_{A}^{\prime}\right) \geq \delta$, it follows that $M\left(\Gamma_{A}\right)>\varepsilon$.

Then $f$ is $K$-quasiconformal, with

$$
\left.K(\varepsilon, \delta, n)=\left(\exp \left(\omega_{n-1} \cdot \varepsilon^{-1}\right)^{1 /(n-1)}\right) / g(\delta)\right)^{n-1} .
$$

Theorem 4. Let $f: D \rightarrow D^{\prime}$ be such that there exists $\delta>0$ such that for every path family $\Gamma$ from $D$ such that $M(\Gamma)=\infty$, it results that $M\left(\Gamma^{\prime}\right)>\delta$. Then $f$ is quasiconformal.

Proof. Suppose that $H(\cdot, f)$ is an unbounded function on $D$. Then there exists $x_{m} \in D$ such that $x_{m} \neq \infty, f\left(x_{m}\right) \neq \infty, H\left(x_{m}, f\right)>\exp \left(2^{m}\right)$. Let $y_{m}=f\left(x_{m}\right)$ for every $m \in N$. Then we can find $r_{m}>0$ such that $L\left(x_{m}, f, r_{m}\right)>$ $\exp \left(2^{m}\right) l\left(x_{m}, f, r_{m}\right)$ and $\bar{B}\left(y_{m}, f, r_{m}\right) \subset D^{\prime}$ for every $m \in N$. We abbreviate $L\left(x_{m}, f, r_{m}\right)$ to $L_{m}$ and $l\left(x_{m}, f, r_{m}\right)$ to $l_{m}$ and let $B_{m}=f^{-1}\left(B\left(y_{m}, l_{m}\right)\right), C_{m}=$ $C f^{-1}\left(B\left(y_{m}, L_{m}\right)\right), A_{m}=R\left(B_{m}, C_{m}\right)$ for every $m \in N$. We remark that we can choose the rings $A_{m}$ such that the path families $\Gamma_{A_{m}}$ are separate. Then $M\left(\Gamma_{A_{m}}\right) \geq h(1)$ and

$$
M\left(\Gamma_{A_{m}}^{\prime}\right)=\omega_{n-1}\left(\log \left(\frac{L_{m}}{l_{m}}\right)\right)^{n-1} \leq \omega_{n-1} \log \left(\exp \left(2^{m}\right)\right)^{1-n} \leq \omega_{n-1} 2^{-m}
$$

for every $m \in N$. Let $p \in N$ be such that $\omega_{n-1} \sum_{m \geq p} 2^{-m}<\delta$. If we take $\Gamma=\bigcup_{m \geq p} \Gamma_{A_{m}}$ and $\Gamma^{\prime}=\bigcup_{m \geq p} \Gamma_{A_{m}}^{\prime}$, we see that $M(\Gamma)=\sum_{m \geq p} M\left(\Gamma_{A_{m}}\right)=\infty$ and $M\left(\Gamma^{\prime}\right)=\sum_{m \geq p} M\left(\Gamma_{A_{m}}^{\prime}\right)<\delta$, which represents a contradiction. 
Remark 1. The referee pointed out to me that we can replace Condition a) of Theorem 2 with a weaker condition, i.e., "There exist $\varepsilon>0$ and $\delta>0$ such that $M\left(\Gamma_{A}^{\prime}\right)<\delta$ for every ring $A$ in $D$ such that $M\left(\Gamma_{A}\right)=\varepsilon "$. This condition will imply Condition a). Indeed, assume that $A$ is a ring in $D$ such that $M\left(\Gamma_{A}\right)<\varepsilon$. Then there exists a ring $A_{1}$ separating the boundary components of $A$ such that $M\left(\Gamma_{A_{1}}\right)=\varepsilon$. If we assume that $M\left(\Gamma_{A_{1}}^{\prime}\right)<\delta$, then $M\left(\Gamma_{A}\right) \leq M\left(\Gamma_{A_{1}}^{\prime}\right)<\delta$. Also, we can replace Condition a) of Theorem 3 with the following weaker condition: "There exists $\varepsilon>0$ and $\delta>0$ such that $M\left(\Gamma_{A}^{\prime}\right)>\varepsilon$ for every ring $A$ in $D$ such that $M\left(\Gamma_{A}\right)=\delta$ "; the proof is the same. From this remark we immediately get the following corollary:

Corollary 1. Let $f: D \rightarrow D^{\prime}$ be such that there exists $\alpha>0$ and $\gamma:(0, \alpha] \rightarrow$ $(0, \infty)$ such that $M\left(\Gamma_{A}^{\prime}\right) \leq \gamma\left(M\left(\Gamma_{A}\right)\right)$ for every ring $A$ in $D$ such that $0<$ $M\left(\Gamma_{A}\right) \leq \alpha$. Then $f$ is $K$-quasiconformal with $K=K(\alpha, \gamma, n)$.

We remark that if $f: D \rightarrow D^{\prime}$ is $K$-quasiconformal, then for every ring $A$ in $D, M\left(\Gamma_{A}^{\prime}\right) \leq K M\left(\Gamma_{A}\right)$; hence the condition of Corollary 1 is satisfied for $\gamma:(0, \infty) \rightarrow(0, \infty), \gamma(x)=K x$. Our result shows that we can have quasiconformality for an arbitrary function $\gamma$.

\section{References}

[1] Caraman, P.: Characterization of the quasiconformality by arc families of extremal length zero. - Ann. Acad. Sci. Fenn. Ser. A I Math. 528, 1973, 1-10.

[2] VÄISÄLÄ, J.: Lectures on $n$-dimensional quasiconformal mappings. - Springer-Verlag, Berlin-Heidelberg-New York, 1971.

Academy of Sciences

Institute of Mathematics

Str. Academiei 14

R-70109 Bucharest

Romania

Received 17 October 1988 